RASĀYAN J. Chem.

Vol. 13 | No. 4 |2352-2360| October - December | 2020 ISSN: 0974-1496 | e-ISSN: 0976-0083 | CODEN: RJCABP

\title{
SYNTHESIS OF 1,1,2-TRISUBSTITUTED CYCLOPROPANE RING THROUGH CARBON-CARBON BOND NUCLEOPHILE- INTERCEPTED BECKMANN FRAGMENTATION REACTION
}

\author{
Nandkishor Chandan \\ Department of Chemistry, Siddharth College of Arts, Science and Commerce, \\ Fort Mumbai-400 001, Maharashtra, India \\ E-mail: nandkishorc@gmail.com
}

\begin{abstract}
The Beckmann fragmentation reaction, considered to be a special type of the Beckmann rearrangement only occurs in particular when $\alpha$-carbon of the oxime is substituted with alkyl or aryl groups which are capable of stabilizing a carbocation formed when a nitrile group results. In this project, the oxime of dialkyl 2-(3-oxocyclohexyl)malonate are undergone derivatization with $p$-toluenesulfonyl chloride or 2,4-dinitrofluorobenzene. The tosyloxime or oxime ether with no substituents at $\alpha$-carbon of the oxime derivatives was on treatment with base $\mathrm{NaH}$ or $\mathrm{CsCO}_{3}$ initiates the Beckmann fragmentation, wherein this reaction the carbocation formed on $\alpha$-carbon atom simultaneously attacked by dialky malonate carbon as a nucleophile in the same basic condition and results in the carbon-carbon bond formation as to substituted cyclopropane rings. The strained substituted cyclopropane rings occurred in many complex natural products including terpenoids, steroids, alkaloids pheromones, fatty acid metabolites and unusual amino acids. Some Natural products with cyclopropane motifs not only furnished fascinating structures, but also exhibited versatile biological activities, such as cytotoxic, anti-HIV, antimicrobial, antiviral, and immunosuppressive effects.
\end{abstract}

Keywords: Oxime, Oxime Ether, Tosyloxime, Cyclopropane, Beckmann Fragmentation etc.

(C) RASĀYAN. All rights reserved

\section{INTRODUCTION}

The intermolecular cyclopropanation reactions were reported by many researchers wherein olefins are treated with activated methylene in presence of heavy metal as catalyst ${ }^{1}$. The Beckmann fragmentation through the $\mathrm{C}-\mathrm{C}$ bond breaking and $\mathrm{C}-\mathrm{C}$ bond making leads to the intramolecular cyclopropanation with a very good yield to 1,1,2-trisubstituted cyclopropane ring without the use of any heavy metals as a catalyst. The concept of cyclopropanation was an unintended result, ${ }^{2-5}$, when we tried to synthesized antiBredt's imine compounds as listed in Fig.-1. According to Bredt's rule ${ }^{6}$ in the carbon bicyclic system, carbon-carbon double bonds at the bridgeheads cannot exist for the small ring size. ${ }^{7}$ But, there are reports of anti-Bredt's rule compounds, ${ }^{8-12}$ wherein the bicyclic compound has an unsaturated center at the bridgehead position, although isolation of these compounds was limited. ${ }^{13}$ The examples of bicyclic imines, in which carbon-nitrogen double bond having the similar geometry to its olefinic analog are also reported and these anti-Bredt imine compounds have been isolated including A, B and C ${ }^{14}$ (Fig.-1)

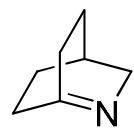

A<smiles>C1=NCC2CCC1C2</smiles>

B

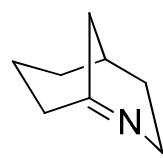

C

Fig.-1: Anti-Bredt Imines ${ }^{14}$

The methodology ${ }^{3}$ for the synthesis of 2,2,5-trisubstituted pyrrolidine was already developed and the same concept was applied to synthesize the anti-Bred't compound 4 (Scheme-1). In this methodology, it was thought that when tosyloxime $\mathbf{3}$ or oxime ether when treated with base the malonate group can act as

Rasayan J. Chem., 13(4), 2352-2360(2020)

http://dx.doi.org/10.31788/ RJC.2020.1345745 
RASĀYAN $J$. Chem.

Vol. 13 | No. 4 |2352-2360| October - December | 2020

nucleophilic carbon in the basic condition and tosyl group as good leaving group makes nitrogen atom as electrophile leads to ring closure as anticipated anti-Bredt's compound 4 (Scheme 1) but instead of the anti-Bredt compound 4, the Beckmann fragmentation ${ }^{15-17}$ product 5 was obtained in a high yield of $74 \%$ (Scheme-2). The malonate nucleophilic carbon attacked on $\alpha$-carbon atom instead of the nitrogen atom of the tosyl oxime and this resulted in the formation of the Beckmann fragmentation product as a substituted

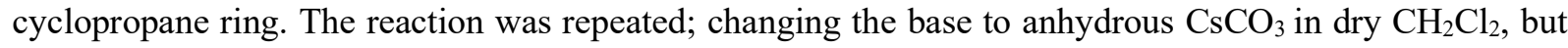
gave the same results and no conditions which gave the anti-Bredt product could be identified. The cyclopropane ring is a highly strained system but preferred instead of the five-membered ring system confirmed by the ${ }^{1} \mathrm{H}$ NMR and mass spectra (Scheme 2). The same Beckmann fragmentation was exploited for various substitutions with changing the active methylene group as dialkyl malonate, ethyl 2cyanoacetate and ethyl 2-nitroethanoate.<smiles>CCOC(=O)C1CCCCN(C(=O)OCC)C(=O)CCC1</smiles>

3

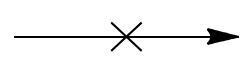

d

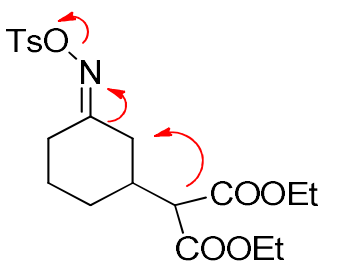

3

Reagents and conditions (d) i) $\mathrm{NaH}$, dry THF, reflux, 30 min; ii) anhydrous $\mathrm{CsCO}_{3}, \mathrm{CH}_{2} \mathrm{Cl}_{2}$, reflux.

Scheme-1: Synthesis of Anti-Bredt Compound<smiles>CCOC(=O)C1(C(=O)OCC)N=C2CCC1C2</smiles>

Anti-Bredt 4

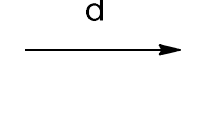

Reagents and conditions (d) i) $\mathrm{NaH}$, dry THF, reflux, 30 min; ii) anhydrous $\mathrm{CsCO}_{3}, \mathrm{CH}_{2} \mathrm{Cl}_{2}$, reflux. Scheme-2: Cyclopropanation Reaction

\section{EXPERIMENTAL}

\section{General Procedure for the Preparation of Michael Adduct}

The $7.50 \mathrm{mmol}$ of cyclohex-2-enone $(0.72 \mathrm{ml})$ was added drop-wise to a well-stirred mixture of 6.25 mmol Diethyl malonate $(0.95 \mathrm{ml})$ and $9.37 \mathrm{mmol}$ of anhydrous $\mathrm{K}_{2} \mathrm{CO}_{3}(1.29 \mathrm{~g})$ in dry $\mathrm{CH}_{2} \mathrm{Cl}_{2} 20 \mathrm{ml}$ and the mixture was stirred for $15 \mathrm{hr}$ at r.t. or till completion of the reaction by $\mathrm{TLC}^{18}$. Water and EtOAc were added sequentially and the organic layers were separated, washed with $1 \mathrm{M} \mathrm{HCl}$, water, brine and dried over $\mathrm{Na}_{2} \mathrm{SO}_{4}$. The concentration of the organic layer gave the crude product, which was purified by flash column chromatography (eluting with EtOAc : petrol) to afford the Michael adduct product 1.

\section{General Procedure for Preparation Oxime of Michael Adduct}

To the solution of $3.90 \mathrm{mmol}$ Michael adduct ketone $1(1 \mathrm{~g})$ in $20 \mathrm{ml}$ EtOH has added $7.81 \mathrm{mmol}$ of $\mathrm{NH}_{2} \mathrm{OH} \cdot \mathrm{HCl}(531 \mathrm{mg})$ and $9.75 \mathrm{mmol}$ of $\mathrm{Et}_{3} \mathrm{~N}(984 \mathrm{mg})$ and the reaction mixture was heated to reflux for $2 \mathrm{hr}$. The progress of the reaction was monitored by TLC and on completion of the reaction mixture was allowed to cool to room temperature ${ }^{19}$. EtOH was removed under reduced pressure and the residue was dissolved in EtOAc and $\mathrm{H}_{2} \mathrm{O}$ added. The organic layer was separated, washed with brine and dried over $\mathrm{Na}_{2} \mathrm{SO}_{4}$. The concentration of the organic layer gave the crude product, which was purified by flash column chromatography (eluting with EtOAc : petrol) to afford the oxime product 2.

\section{General Procedure for Preparation Tosyl Oxime From Oxime}

The stirring solution $3.69 \mathrm{mmol}$ of 2 oxime $(1 \mathrm{~g})$ in dry $20 \mathrm{ml} \mathrm{CH}_{2} \mathrm{Cl}_{2}$ at $0{ }^{\circ} \mathrm{C}$ was treated with $7.38 \mathrm{mmol}$ of $\mathrm{Et}_{3} \mathrm{~N}$ or pyridine $(745 \mathrm{mg}$ or $583 \mathrm{mg}$ ) followed by slow addition $5.53 \mathrm{mmol}$ of $p$-toluenesulphonyl 
RASĀYAN J. Chem.

Vol. 13 | No. 4 |2352-2360| October - December | 2020

chloride $(1.05 \mathrm{~g})$. The reaction was further stirred for $2 \mathrm{hr}$ and progress was monitored by TLC and after completion of the reaction, $(1 \mathrm{M}) \mathrm{HCl}$ was added and the product was extracted by $\mathrm{CH}_{2} \mathrm{Cl}_{2}$ and the extracts were washed with saturated $\mathrm{NaHCO}_{3}$ and dried over $\mathrm{Na}_{2} \mathrm{SO}_{4}{ }^{19}$. The concentration of $\mathrm{CH}_{2} \mathrm{Cl}_{2}$ gave the crude product which was purified by flash column chromatography (eluting with EtOAc : petrol) to afford the tosyl oxime product 3 .

\section{General Procedure for Cyclopropanation through Beckmann Fragmentation ${ }^{20}$}

To the solution, $2.35 \mathrm{mmol}$ of compound 3 tosyl oxime (1g) in dry THF at room temperature, $7.05 \mathrm{mmol}$ of $\mathrm{NaH}(169 \mathrm{mg}, 60 \%$ dispersion in mineral oil) was added and heated to reflux for $30 \mathrm{~min}$ to $1 \mathrm{hr}$ and the progress of the reaction was monitored by $\mathrm{TLC}^{20}$. After completion of the reaction, saturated $\mathrm{NH}_{4} \mathrm{Cl}$ was added and the Beckmann fragmentation product as substituted cyclopropane was extracted by EtOAc. The extracts were dried over $\mathrm{Na}_{2} \mathrm{SO}_{4}$, the concentration of reaction mixture gave the crude product which was purified by flash column chromatography (eluting with EtOAc: petrol) to afford 1,1,2-trisubstituted cyclopropane ring as products 4 .

\section{( \pm )Diethyl 2-(3-oxocyclohexyl)malonate, 1}<smiles>CCOC(=O)C(C(=O)OCC)C1CCCC(=O)C1</smiles>

Following the general procedure, $23.4 \mathrm{mmol}$ of cyclohex-2-enone $(2.26 \mathrm{ml})$ was added to a solution 19.5 mmol of diethyl malonate $(2.97 \mathrm{ml})$ and $29.3 \mathrm{mmol}$ of anhydrous $\mathrm{K}_{2} \mathrm{CO}_{3}(3.92 \mathrm{~g})$ in dry $\mathrm{CH}_{2} \mathrm{Cl}_{2}(30 \mathrm{~mL})$ and the mixture was stirred at r.t. to give crude product which was purified by flash column chromatography to afford adduct $2(4.10 \mathrm{~g}, 82 \%)$ as a colourless oil ${ }^{21} ; \mathrm{R}_{f}=0.53$ (EtOAc : petrol, 4:6); $v_{\max }($ film $) / \mathrm{cm}^{-1} 2982,1731,1448,1423,1297,1229,1156,1061,965 ; \delta_{\mathrm{H}}\left(400 \mathrm{MHz} ; \mathrm{CDCl}_{3} ; \mathrm{Me}_{4} \mathrm{Si}\right) 1.23$ $\left(6 \mathrm{H}, \mathrm{t}, J 7.1 \mathrm{~Hz}, 2 \times \mathrm{OCH}_{2} \mathrm{CH}_{3}\right), 1.47,1.65\left(2 \mathrm{H}, \mathrm{m}, \mathrm{COCH}_{2} \mathrm{CH}_{2} \mathrm{CH}_{2}\right), 1.90\left(2 \mathrm{H}, \mathrm{m}, \mathrm{COCH}_{2} \mathrm{CH}_{2} \mathrm{CH}_{2}\right)$, $2.23\left(2 \mathrm{H}, \mathrm{m}, \mathrm{COCH}_{2} \mathrm{CH}_{2} \mathrm{CH}_{2}\right), 2.39\left(2 \mathrm{H}, \mathrm{m}, \mathrm{COCH}_{2} \mathrm{CH}\right), 2.49\left(1 \mathrm{H}, \mathrm{m}, \mathrm{COCH}_{2} \mathrm{CH}\right), 3.26(1 \mathrm{H}, \mathrm{d}, J 7.9 \mathrm{~Hz}$ $\left.\mathrm{CH}(\mathrm{COOEt})_{2}\right), 4.17\left(4 \mathrm{H}, \mathrm{q}, J 7.1 \mathrm{~Hz}, 2 \times \mathrm{OCH}_{2} \mathrm{CH}_{3}\right) ; \delta_{\mathrm{C}}\left(100 \mathrm{MHz} ; \mathrm{CDCl}_{3} ; \mathrm{Me}_{4} \mathrm{Si}\right) 13.5\left(\mathrm{OCH}_{2} \mathrm{CH}_{3}\right), 23.9$ $\left(\mathrm{COCH}_{2} \mathrm{CH}_{2} \mathrm{CH}_{2}\right), 26.2\left(\mathrm{COCH}_{2} \mathrm{CH}_{2} \mathrm{CH}_{2}\right), 37.4\left(\mathrm{COCH}_{2} \mathrm{CH}\right), 40.4\left(\mathrm{COCH}_{2} \mathrm{CH}_{2} \mathrm{CH}_{2}\right), 44.5\left(\mathrm{COCH}_{2} \mathrm{CH}\right)$, $56.3\left(\mathrm{CH}(\mathrm{COOEt})_{2}\right), 60.9\left(\mathrm{OCH}_{2} \mathrm{CH}_{3}\right), 167.2,167.3(2 \times \mathrm{COO}), 209.1(\mathrm{CO}) ; \mathrm{m} / \mathrm{z}\left(\mathrm{ESI}^{+}\right) 535\left([2 \mathrm{M}+\mathrm{Na}]^{+}\right.$, $85 \%), 279\left([\mathrm{M}+\mathrm{Na}]^{+}, 60 \%\right), 255([\mathrm{M}-\mathrm{H}]-100 \%), \mathrm{HRMS}\left(\mathrm{ESI}^{+}\right) \mathrm{C}_{13} \mathrm{H}_{20} \mathrm{NaO}_{5}^{+}\left([\mathrm{M}+\mathrm{Na}]^{+}\right)$requires 279.1203 ; found 279.1199 .

( \pm )Diethyl 2-(3-(hydroxyimino)cyclohexyl)malonate, 2<smiles>CCOC(=O)C(C(=O)OCC)C1CCCC(=NO)C1</smiles>

Following the general procedure, to the solution $14.9 \mathrm{mmol}$ of adduct $1(3.82 \mathrm{~g})$ in EtOH $(40 \mathrm{ml}), 22.4$ mmol of $\mathrm{NH}_{2} \mathrm{OH} . \mathrm{HCl}(1.54 \mathrm{~g})$ and $29.8 \mathrm{mmol}$ of $\mathrm{Et}_{3} \mathrm{~N}(3.01 \mathrm{~g})$ was added and heated to reflux for $1 \mathrm{hr}$ to give crude product which was purified by flash column chromatography to afford oxime 2 (3.82 g, 94\%) as a colourless oil; $\mathrm{R}_{f}=0.45,0.37$ (EtOAc : petrol, 4:6); $v_{\max }\left(\right.$ film) $/ \mathrm{cm}^{-1} 3240,2938,1731,1448,1369$, 1233, 1156, 1096, 966, 862; (major isomer) $\delta_{\mathrm{H}}\left(400 \mathrm{MHz} ; \mathrm{CDCl}_{3}\right.$; $\left.\mathrm{Me}_{4} \mathrm{Si}\right) 1.17(6 \mathrm{H}, \mathrm{t}, J 7.1 \mathrm{~Hz}, 2 \times$ $\left.\mathrm{OCH}_{2} \mathrm{CH}_{3}\right), 1.34\left(2 \mathrm{H}, \mathrm{m}, \mathrm{CCH}_{2} \mathrm{CH}_{2} \mathrm{CH}_{2}\right), 1.70\left(2 \mathrm{H}, \mathrm{m}, \mathrm{CCH}_{2} \mathrm{CH}_{2} \mathrm{CH}_{2}\right), 1.93\left(2 \mathrm{H}, \mathrm{m}, \mathrm{CCH}_{2} \mathrm{CH}_{2} \mathrm{CH}_{2}\right)$, $2.26\left(2 \mathrm{H}, \mathrm{m}, \mathrm{CCH}_{2} \mathrm{CH}\right), 3.11\left(1 \mathrm{H}, \mathrm{m}, \mathrm{CCH}_{2} \mathrm{CH}\right), 3.19\left(1 \mathrm{H}, \mathrm{d}, J 8.3 \mathrm{~Hz} \mathrm{CH}(\mathrm{COOEt})_{2}\right), 4.11(4 \mathrm{H}, \mathrm{q}, J 7.1$ $\left.\mathrm{Hz}, 2 \times \mathrm{OCH}_{2} \mathrm{CH}_{3}\right), 9.40(1 \mathrm{H}, \mathrm{bs}, \mathrm{OH}) ; \delta_{\mathrm{C}}\left(100 \mathrm{MHz} ; \mathrm{CDCl}_{3} ; \mathrm{Me}_{4} \mathrm{Si}\right) 14.0\left(\mathrm{OCH}_{2} \mathrm{CH}_{3}\right), 23.7,23.8$ $\left(\mathrm{CCH}_{2} \mathrm{CH}_{2} \mathrm{CH}_{2}\right), 25.1,27.8\left(\mathrm{CCH}_{2} \mathrm{CH}_{2} \mathrm{CH}_{2}\right), 29.4,29.5\left(\mathrm{CCH}_{2} \mathrm{CH}_{2} \mathrm{CH}_{2}\right)$, 31.6, $35.5\left(\mathrm{CCH}_{2} \mathrm{CH}\right), 36.7,37.7$ 
RASĀYAN J. Chem.

Vol. 13 | No. 4 |2352-2360| October - December | 2020

$\left(\mathrm{CCH}_{2} \mathrm{CH}\right), 56.9,57.0\left(\mathrm{CH}(\mathrm{COOEt})_{2}\right), 61.3,61.4\left(\mathrm{OCH}_{2} \mathrm{CH}_{3}\right), 158.4,158.7(\mathrm{C}=\mathrm{NOH}), 168.0,168.1$, $168.2(2 \times \mathrm{COO}) ; \mathrm{m} / \mathrm{z}\left(\mathrm{ESI}^{+}\right) 565\left([2 \mathrm{M}+\mathrm{Na}]^{+}, 100 \%\right), 294\left([\mathrm{M}+\mathrm{Na}]^{+}, 50 \%\right), 270([\mathrm{M}-\mathrm{H}]$ ], 100\%), HRMS $\left(\mathrm{ESI}^{+}\right) \mathrm{C}_{13} \mathrm{H}_{21} \mathrm{NNaO}_{5}^{+}\left([\mathrm{M}+\mathrm{Na}]^{+}\right)$requires 294.1312; found 294.1310.

\section{( \pm )Diethyl 2-(3-(tosyloxyimino)cyclohexyl)malonate, 3}<smiles>CCOC(=O)C(C(=O)OCC)C1CCCC(=N)C1</smiles>

Following the general procedure, to the solution $5.09 \mathrm{mmol}$ of oxime $2(1.38 \mathrm{~g})$ in dry $\mathrm{CH}_{2} \mathrm{Cl}_{2}(25 \mathrm{ml})$ was added $10.2 \mathrm{mmol}$ of $\mathrm{Et}_{3} \mathrm{~N}(1.02 \mathrm{~g})$ at $0^{\circ} \mathrm{C}$ followed by $7.63 \mathrm{mmol} p$-toluenesulphonyl chloride $(1.45 \mathrm{~g})$ and the mixture stirred for $2 \mathrm{hr}$ at r.t. to give crude product which was purified by column chromatography to afford tosyl oxime $3(1.82 \mathrm{~g}, 84 \%)$ as colourless a oil; $\mathrm{R}_{f}=0.59$ (EtOAc : petrol, 4:6); $v_{\max }\left(\right.$ film) $/ \mathrm{cm}^{-1}$ 2938, 1730, 1447, 1369, 1155, 1032, 817, 732; (major isomer) $\delta_{\mathrm{H}}\left(400 \mathrm{MHz} ; \mathrm{CDCl}_{3} ; \mathrm{Me}_{4} \mathrm{Si}\right) 1.23(6 \mathrm{H}, \mathrm{t}, J$ $\left.7.1 \mathrm{~Hz}, 2 \times \mathrm{OCH}_{2} \mathrm{CH}_{3}\right), 1.47\left(2 \mathrm{H}, \mathrm{m}, \mathrm{CCH}_{2} \mathrm{CH}_{2} \mathrm{CH}_{2}\right), 1.86\left(2 \mathrm{H}, \mathrm{m}, \mathrm{CCH}_{2} \mathrm{CH}_{2} \mathrm{CH}_{2}\right), 2.03(2 \mathrm{H}, \mathrm{m}$, $\left.\mathrm{CCH}_{2} \mathrm{CH}_{2} \mathrm{CH}_{2}\right), 2.28\left(2 \mathrm{H}, \mathrm{m}, \mathrm{CCH}_{2} \mathrm{CH}\right), 2.39\left(3 \mathrm{H}, \mathrm{s}, \mathrm{ArCH}_{3}\right), 2.49\left(1 \mathrm{H}, \mathrm{m}, \mathrm{CCH}_{2} \mathrm{CH}\right), 3.26(1 \mathrm{H}, \mathrm{d}, J 7.9$ $\left.\mathrm{Hz} \mathrm{CH}(\mathrm{COOEt})_{2}\right), 4.16\left(4 \mathrm{H}, \mathrm{q}, J 7.1 \mathrm{~Hz}, 2 \times \mathrm{OCH}_{2} \mathrm{CH}_{3}\right), 7.29(2 \mathrm{H}, \mathrm{d}, J 8.1 \mathrm{~Hz}, \mathrm{Ar} H), 7.79(2 \mathrm{H}, \mathrm{d}, J 8.1$ $\mathrm{Hz}, \mathrm{Ar} H) ; \delta_{\mathrm{C}}\left(100 \mathrm{MHz} ; \mathrm{CDCl}_{3} ; \mathrm{Me} 4 \mathrm{Si}\right) 13.5\left(\mathrm{OCH}_{2} \mathrm{CH}_{3}\right), 21.1\left(\mathrm{ArCH}_{3}\right), 23.9\left(\mathrm{CCH}_{2} \mathrm{CH}_{2} \mathrm{CH}_{2}\right), 28.2$ $\left(\mathrm{CCH}_{2} \mathrm{CH}_{2} \mathrm{CH}_{2}\right), 37.4\left(\mathrm{CCH}_{2} \mathrm{CH}\right), 40.4\left(\mathrm{CCH}_{2} \mathrm{CH}_{2} \mathrm{CH}_{2}\right), 44.5\left(\mathrm{CCH}_{2} \mathrm{CH}\right), 56.3\left(\mathrm{CH}(\mathrm{COOEt})_{2}\right), 60.9$ $\left(\mathrm{OCH}_{2} \mathrm{CH}_{3}\right), 128.1,128.9,132.1,144.2(\mathrm{ArC}), 167.1$ ( $\left.C=\mathrm{NOTs}\right), 167.3,167.5(2 \times \mathrm{COO}) ; \mathrm{m} / \mathrm{z}\left(\mathrm{ESI}^{+}\right) 873$ $\left([2 \mathrm{M}+\mathrm{Na}]^{+}, 100 \%\right), 448\left([\mathrm{M}+\mathrm{Na}]^{+}, 80 \%\right), \mathrm{HRMS}\left(\mathrm{ESI}^{+}\right) \mathrm{C}_{20} \mathrm{H}_{27} \mathrm{NNaO}_{7} \mathrm{~S}^{+}\left([\mathrm{M}+\mathrm{Na}]^{+}\right)$requires 448.1400; found 448.1400 .

\section{( \pm )Diethyl 2-(3-cyanopropyl)cyclopropane-1,1-dicarboxylate, 5}

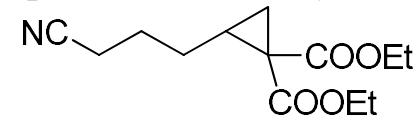

Following the general procedure, to the solution $0.60 \mathrm{mmol}$ of tosyl oxime $3(256 \mathrm{mg})$ in dry THF (10 $\mathrm{ml})$ was added $1.80 \mathrm{mmol}$ of $\mathrm{NaH}$ (43 mg, $60 \%$ dispersion in mineral oil) and heated to reflux for $30 \mathrm{~min}$, instead ring closure pyrroline as bicyclic anti-Bredt's compound, we have isolated Beckman fragmentation product which was purified by flash column chromatography to afford substituted cyclopropane $5(113 \mathrm{mg}, 74 \%)$ as a colourless oil; $\mathrm{R}_{f}=0.49$ (EtOAc : petrol, $\left.4: 6\right)^{22,23} ; v_{\max }($ film $) / \mathrm{cm}^{-1}$ 2938, 2256, 1731, 1431, 1447, 1369, 1156, 1096, 1030, 967, 916, 862; $\delta_{\mathrm{H}}\left(400 \mathrm{MHz} ; \mathrm{CDCl}_{3} ; \mathrm{Me}_{4} \mathrm{Si}\right) 1.24$, $1.27\left(6 \mathrm{H}, \mathrm{t}, J 7.1 \mathrm{~Hz}, 2 \times \mathrm{OCH}_{2} \mathrm{CH}_{3}\right), 1.33\left(2 \mathrm{H}, \mathrm{m}, \mathrm{NCCH}_{2} \mathrm{CH}_{2} \mathrm{CH}_{2}\right), 1.39(1 \mathrm{H}, \mathrm{m}, \mathrm{CHCHHC}), 1.55(1 \mathrm{H}$, $\left.\mathrm{m}, \mathrm{CHCH}_{2} \mathrm{C}\right), 1.74(1 \mathrm{H}, \mathrm{m}, \mathrm{CHCHHC}), 1.82\left(2 \mathrm{H}, \mathrm{m}, \mathrm{NCCH}_{2} \mathrm{CH}_{2} \mathrm{CH}_{2}\right), 2.33(2 \mathrm{H}, \mathrm{t}, J 7.1 \mathrm{~Hz}$ $\left.\mathrm{NCCH}_{2} \mathrm{CH}_{2} \mathrm{CH}_{2}\right), 4.11,4.21\left(4 \mathrm{H}, \mathrm{q}, J 7.1 \mathrm{~Hz}, 2 \times \mathrm{OCH}_{2} \mathrm{CH}_{3}\right) ; \delta_{\mathrm{C}}\left(100 \mathrm{MHz} ; \mathrm{CDCl}_{3} ; \mathrm{Me}_{4} \mathrm{Si}\right) 13.8$ $\left(\mathrm{OCH}_{2} \mathrm{CH}_{3}\right), 16.5\left(\mathrm{NCCH}_{2} \mathrm{CH}_{2} \mathrm{CH}_{2}\right), 20.3\left(\mathrm{CHCH}_{2} \mathrm{C}\right), 24.5\left(\mathrm{NCCH}_{2} \mathrm{CH}_{2} \mathrm{CH}_{2}\right), 26.4\left(\mathrm{CHCH}_{2} \mathrm{C}\right), 27.3$ $\left(\mathrm{NCCH}_{2} \mathrm{CH}_{2} \mathrm{CH}_{2}\right), 33.8\left(\mathrm{C}(\mathrm{COOEt})_{2}\right), 61.3\left(\mathrm{OCH}_{2} \mathrm{CH}_{3}\right), 118.9(\mathrm{CN}), 167.6,169.8(2 \times \mathrm{COO}) ; \mathrm{m} / \mathrm{z}\left(\mathrm{ESI}^{+}\right)$ $276\left([\mathrm{M}+\mathrm{Na}]^{+}, 100 \%\right), \mathrm{HRMS}\left(\mathrm{ESI}^{+}\right) \mathrm{C}_{13} \mathrm{H}_{19} \mathrm{NNaO}_{4}^{+}\left([\mathrm{M}+\mathrm{Na}]^{+}\right)$requires 276.1206; found 276.1207.

(土)1-ethyl 1-methyl 2-(3-cyanopropyl)cyclopropane-1,1-dicarboxylate, 9

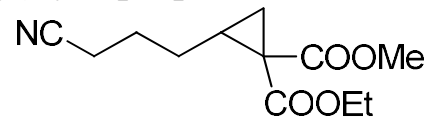

Following the general procedure, to the solution $0.29 \mathrm{mmol}$ of tosyl oxime $\mathbf{8}(120 \mathrm{mg})$ in dry THF $(5 \mathrm{ml})$ was added $0.87 \mathrm{mmol}$ of $\mathrm{NaH}(21 \mathrm{mg}, 60 \%$ dispersion in mineral oil) and heated to reflux for $30 \mathrm{~min}$, cyclopropanation product was isolated as competing Beckman fragmentation reaction which was purified by flash column chromatography to afford substituted cyclopropane $9(47 \mathrm{mg}, 69 \%)$ as a colourless oil; $\mathrm{R}_{f}$ 2355 
$=0.48$ (EtOAc : petrol, 4:6); $v_{\max }\left(\right.$ film) $/ \mathrm{cm}^{-1}$ 2939, 2255, 1730,1732, 1431, 1448, 1369, 1180, 1159. $\delta_{\mathrm{H}}\left(400 \mathrm{MHz} ; \mathrm{CDCl}_{3} ; \mathrm{Me}_{4} \mathrm{Si}\right) 1.25\left(3 \mathrm{H}, \mathrm{t}, J 7.1 \mathrm{~Hz}, \mathrm{OCH}_{2} \mathrm{CH}_{3}\right), 1.33\left(2 \mathrm{H}, \mathrm{m}, \mathrm{NCCH}_{2} \mathrm{CH}_{2} \mathrm{CH}_{2}\right), 1.38(1 \mathrm{H}$, $\mathrm{m}, \mathrm{CHCH} H \mathrm{C}), 1.57\left(1 \mathrm{H}, \mathrm{m}, \mathrm{CHCH}_{2} \mathrm{C}\right), 1.75(1 \mathrm{H}, \mathrm{m}, \mathrm{CHCHHC}), 1.82\left(2 \mathrm{H}, \mathrm{m}, \mathrm{NCCH}_{2} \mathrm{CH}_{2} \mathrm{CH}_{2}\right), 2.33$ $\left(2 \mathrm{H}, \mathrm{t}, J 7.0 \mathrm{~Hz} \mathrm{NCCH} \mathrm{CH}_{2} \mathrm{CH}_{2}\right), 4.20\left(2 \mathrm{H}, \mathrm{q}, J 7.1 \mathrm{~Hz}, \mathrm{OCH}_{2} \mathrm{CH}_{3}\right) ; 4.09\left(3 \mathrm{H}, \mathrm{OCH}_{3}\right) \delta_{\mathrm{C}}(100 \mathrm{MHz}$; $\left.\mathrm{CDCl}_{3} ; \mathrm{Me} 4 \mathrm{Si}\right) 13.9\left(\mathrm{OCH}_{2} \mathrm{CH}_{3}\right), 16.5\left(\mathrm{NCCH}_{2} \mathrm{CH}_{2} \mathrm{CH}_{2}\right), 20.6\left(\mathrm{CHCH}_{2} \mathrm{C}\right), 24.2\left(\mathrm{NCCH}_{2} \mathrm{CH}_{2} \mathrm{CH}_{2}\right), 26.4$ $\left(\mathrm{CHCH}_{2} \mathrm{C}\right), 27.3\left(\mathrm{NCCH}_{2} \mathrm{CH}_{2} \mathrm{CH}_{2}\right), 33.9\left(\mathrm{C}(\mathrm{COOR})_{2}\right), 60.1\left(\mathrm{OCH}_{3}\right), 61.3\left(\mathrm{OCH}_{2} \mathrm{CH}_{3}\right), 118.9(\mathrm{CN})$, $167.5,169.9(2 \times \mathrm{COO}) ; \mathrm{m} / \mathrm{z}\left(\mathrm{ESI}^{+}\right) 262\left([\mathrm{M}+\mathrm{Na}]^{+}, 100 \%\right), \mathrm{HRMS}\left(\mathrm{ESI}^{+}\right) \mathrm{C}_{12} \mathrm{H}_{17} \mathrm{NNaO}_{4}^{+}\left([\mathrm{M}+\mathrm{Na}]^{+}\right)$ requires 262.1055; found 262.1109 .

\section{( \pm 1-tert-Butyl 1-ethyl 2-(3-cyanopropyl)cyclopropane-1,1-dicarboxylate, 13}

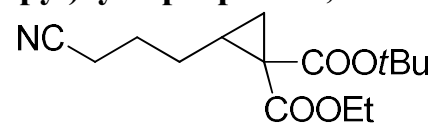

Following the general procedure, to the solution $0.30 \mathrm{mmol}$ of tosyl oxime $12(136 \mathrm{mg})$ in dry THF (5ml) was added $0.90 \mathrm{mmol}$ of $\mathrm{NaH}(21 \mathrm{mg}, 60 \%$ dispersion in mineral oil) and heated to reflux for $30 \mathrm{~min}$, cyclopropanation product was isolated as competing Beckman fragmentation reaction which was purified by flash column chromatography to afford substituted cyclopropane $\mathbf{1 3}(49 \mathrm{mg}, 58 \%)$ as a colourless oil; $\mathrm{R}_{f}=0.50$ (EtOAc : petrol, 4:6); $v_{\max }\left(\right.$ film) $/ \mathrm{cm}^{-1} 2935,2250,1735,1739,1432,1449,1369,1194,1158$. $\delta_{\mathrm{H}}\left(400 \mathrm{MHz} ; \mathrm{CDCl}_{3} ; \mathrm{Me}_{4} \mathrm{Si}\right) 1.1(9 \mathrm{H}, \mathrm{s}, t \mathrm{Bu}), 1.27\left(3 \mathrm{H}, \mathrm{t}, J 7.0 \mathrm{~Hz}, \mathrm{OCH}_{2} \mathrm{CH}_{3}\right), 1.33(2 \mathrm{H}, \mathrm{m}$, $\left.\mathrm{NCCH}_{2} \mathrm{CH}_{2} \mathrm{CH}_{2}\right), 1.33(1 \mathrm{H}, \mathrm{m}, \mathrm{CHCH} H \mathrm{C}), 1.59\left(1 \mathrm{H}, \mathrm{m}, \mathrm{CHCH}_{2} \mathrm{C}\right), 1.75(1 \mathrm{H}, \mathrm{m}, \mathrm{CHCHHC}), 1.82(2 \mathrm{H}$, $\left.\mathrm{m}, \mathrm{NCCH}_{2} \mathrm{CH}_{2} \mathrm{CH}_{2}\right), 2.33\left(2 \mathrm{H}, \mathrm{t}, J 7.0 \mathrm{~Hz} \mathrm{NCCH} \mathrm{CH}_{2} \mathrm{CH}_{2}\right), 4.20\left(2 \mathrm{H}, \mathrm{q}, J 7.0 \mathrm{~Hz}, \mathrm{OCH}_{2} \mathrm{CH}_{3}\right) ; \delta_{\mathrm{C}}(100$ $\left.\mathrm{MHz} ; \mathrm{CDCl}_{3} ; \mathrm{Me} 4 \mathrm{Si}\right) 13.1(t \mathrm{Bu}), 13.7\left(\mathrm{OCH}_{2} \mathrm{CH}_{3}\right), 16.5\left(\mathrm{NCCH}_{2} \mathrm{CH}_{2} \mathrm{CH}_{2}\right), 20.6\left(\mathrm{CHCH}_{2} \mathrm{C}\right), 24.11$ $\left(\mathrm{NCCH}_{2} \mathrm{CH}_{2} \mathrm{CH}_{2}\right), 26.3\left(\mathrm{CHCH}_{2} \mathrm{C}\right), 27.3\left(\mathrm{NCCH}_{2} \mathrm{CH}_{2} \mathrm{CH}_{2}\right), 34.0\left(\mathrm{C}(\mathrm{COOR})_{2}\right), 60.0\left(\mathrm{OCH}_{2} \mathrm{CH}_{3}\right), 63.5$ $\left(\mathrm{OC}\left(\mathrm{CH}_{3}\right)_{3}\right), 118.9(\mathrm{CN}), 167.9,173.3(2 \times \mathrm{COO}) ; \mathrm{m} / \mathrm{z}\left(\mathrm{ESI}^{+}\right) 304\left([\mathrm{M}+\mathrm{Na}]^{+}, 100 \%\right), \mathrm{HRMS}\left(\mathrm{ESI}^{+}\right)$ $\mathrm{C}_{15} \mathrm{H}_{23} \mathrm{NNaO}_{4}^{+}\left([\mathrm{M}+\mathrm{Na}]^{+}\right)$requires 304.1525 ; found 304.1208 .

\section{( \pm 1-Benzyl 1-ethyl 2-(3-cyanopropyl)cyclopropane-1,1-dicarboxylate, 17}<smiles>CCOC(=O)C1(C)C[C@H]1CCCC#N</smiles>

Following the general procedure, to the solution $0.32 \mathrm{mmol}$ of tosyl oxime $\mathbf{1 6}(160 \mathrm{mg})$ in dry THF (5ml) was added $0.98 \mathrm{mmol}$ of $\mathrm{NaH}(23 \mathrm{mg}, 60 \%$ dispersion in mineral oil) and heated to reflux for $30 \mathrm{~min}$, cyclopropanation product was isolated as competing Beckman fragmentation reaction which was purified by flash column chromatography to afford substituted cyclopropane $17(69 \mathrm{mg}, 67 \%)$ as a colourless oil; $\mathrm{R}_{f}=0.51$ (EtOAc : petrol, 4:6); $v_{\max }\left(\right.$ film) $/ \mathrm{cm}^{-1} 3078,2939,2252,1731,1735,1432,1438,1359,1172$, 1157. $\delta_{\mathrm{H}}\left(400 \mathrm{MHz} ; \mathrm{CDCl}_{3} ; \mathrm{Me}_{4} \mathrm{Si}\right) 1.23\left(3 \mathrm{H}, \mathrm{t}, J 7.0 \mathrm{~Hz}, \mathrm{OCH}_{2} \mathrm{CH}_{3}\right), 1.31\left(2 \mathrm{H}, \mathrm{m}, \mathrm{NCCH}_{2} \mathrm{CH}_{2} \mathrm{CH}_{2}\right), 1.38$ $(1 \mathrm{H}, \mathrm{m}, \mathrm{CHCH} H \mathrm{C}), 1.55\left(1 \mathrm{H}, \mathrm{m}, \mathrm{CHCH}_{2} \mathrm{C}\right), 1.78(1 \mathrm{H}, \mathrm{m}, \mathrm{CHCHHC}), 1.82\left(2 \mathrm{H}, \mathrm{m}, \mathrm{NCCH}_{2} \mathrm{CH}_{2} \mathrm{CH}_{2}\right)$, $2.34\left(2 \mathrm{H}, \mathrm{t}, J 7.0 \mathrm{~Hz} \mathrm{NCCH} \mathrm{CH}_{2} \mathrm{CH}_{2}\right), 4.21\left(2 \mathrm{H}, \mathrm{q}, J 7.0 \mathrm{~Hz}, \mathrm{OCH}_{2} \mathrm{CH}_{3}\right) ; 4.38\left(2 \mathrm{H}, \mathrm{OCH}_{2} \mathrm{Ph}\right), 7.2-7.5$ $(5 \mathrm{H}, \mathrm{m}, \mathrm{ArH}) \delta_{\mathrm{C}}\left(100 \mathrm{MHz} ; \mathrm{CDCl}_{3} ; \mathrm{Me}_{4} \mathrm{Si}\right) 13.9\left(\mathrm{OCH}_{2} \mathrm{CH}_{3}\right), 16.5\left(\mathrm{NCCH}_{2} \mathrm{CH}_{2} \mathrm{CH}_{2}\right), 20.76\left(\mathrm{CHCH}_{2} \mathrm{C}\right)$, $24.4\left(\mathrm{NCCH}_{2} \mathrm{CH}_{2} \mathrm{CH}_{2}\right), 26.5\left(\mathrm{CHCH}_{2} \mathrm{C}\right), 27.4\left(\mathrm{NCCH}_{2} \mathrm{CH}_{2} \mathrm{CH}_{2}\right), 33.8\left(\mathrm{C}(\mathrm{COOR})_{2}\right), 61.3\left(\mathrm{OCH}_{2} \mathrm{CH}_{3}\right)$, $60.9\left(\mathrm{OCH}_{2} \mathrm{Ph}\right), 118.9(\mathrm{CN}), 123,125,127,149,(\mathrm{ArC}), 167.7,173.2(2 \times \mathrm{COO}) ; \mathrm{m} / \mathrm{z}\left(\mathrm{ESI}^{+}\right) 338$ $\left([\mathrm{M}+\mathrm{Na}]^{+}, 100 \%\right), \mathrm{HRMS}\left(\mathrm{ESI}^{+}\right) \mathrm{C}_{18} \mathrm{H}_{21} \mathrm{NNaO}_{4}{ }^{+}\left([\mathrm{M}+\mathrm{Na}]^{+}\right)$requires 338.1368; found 338.1203.

( \pm ) Ethyl 1-cyano-2-(3-cyanopropyl)cyclopropanecarboxylate, 25<smiles>CCOC(=O)C1(C#N)CC1CCCC#N</smiles>

Following the general procedure, to the solution $0.24 \mathrm{mmol}$ of tosyl oxime $\mathbf{2 4}(89 \mathrm{mg})$ in dry THF $(5 \mathrm{ml})$ was added $0.70 \mathrm{mmol}$ of $\mathrm{NaH}(16 \mathrm{mg}, 60 \%$ dispersion in mineral oil) and heated to reflux for $30 \mathrm{~min}$, cyclopropanation product was isolated as competing Beckman fragmentation reaction which was purified 
RASĀYAN $J$. Chem.

Vol. 13 | No. 4 |2352-2360| October - December | 2020

by flash column chromatography to afford substituted cyclopropane $\mathbf{2 5}(17 \mathrm{mg}, \mathbf{3 6} \%)$ as a colourless oil; $\mathrm{R}_{f}=0.39$ (EtOAc : petrol, 4:6); $v_{\max }\left(\right.$ film) $/ \mathrm{cm}^{-1} 2937,2254,2210,1733,1433,1436,1357,1157 . \delta_{\mathrm{H}}(400$ $\left.\mathrm{MHz} ; \mathrm{CDCl}_{3} ; \mathrm{Me}_{4} \mathrm{Si}\right) 1.22\left(3 \mathrm{H}, \mathrm{t}, J 7.0 \mathrm{~Hz}, \mathrm{OCH}_{2} \mathrm{CH}_{3}\right), 1.31\left(2 \mathrm{H}, \mathrm{m}, \mathrm{NCCH}_{2} \mathrm{CH}_{2} \mathrm{CH}_{2}\right), 1.43(1 \mathrm{H}, \mathrm{m}$, $\mathrm{CHCHHC}), 1.55\left(1 \mathrm{H}, \mathrm{m}, \mathrm{CHCH}_{2} \mathrm{C}\right), 1.79(1 \mathrm{H}, \mathrm{m}, \mathrm{CHCHHC}), 1.88\left(2 \mathrm{H}, \mathrm{m}, \mathrm{NCCH}_{2} \mathrm{CH}_{2} \mathrm{CH}_{2}\right), 2.35(2 \mathrm{H}, \mathrm{t}$, $\left.J 7.0 \mathrm{~Hz} \mathrm{NCCH} \mathrm{CH}_{2} \mathrm{CH}_{2}\right), 4.20\left(2 \mathrm{H}, \mathrm{q}, J 7.0 \mathrm{~Hz}, \mathrm{OCH}_{2} \mathrm{CH}_{3}\right) ; \delta_{\mathrm{C}}\left(100 \mathrm{MHz} ; \mathrm{CDCl}_{3} ; \mathrm{Me}_{4} \mathrm{Si}\right) 13.8$ $\left(\mathrm{OCH}_{2} \mathrm{CH}_{3}\right), 16.6\left(\mathrm{NCCH}_{2} \mathrm{CH}_{2} \mathrm{CH}_{2}\right), 20.84\left(\mathrm{CHCH}_{2} \mathrm{C}\right), 24.4\left(\mathrm{NCCH}_{2} \mathrm{CH}_{2} \mathrm{CH}_{2}\right), 26.6\left(\mathrm{CHCH}_{2} \mathrm{C}\right), 27.4$ $\left(\mathrm{NCCH}_{2} \mathrm{CH}_{2} \mathrm{CH}_{2}\right), 34.7$ (C(COOR), $61.3\left(\mathrm{OCH}_{2} \mathrm{CH}_{3}\right)$ 118.9, $120.2(\mathrm{CN}), 173.2(\mathrm{COO}) ; \mathrm{m} / \mathrm{z}\left(\mathrm{ESI}^{+}\right) 229$ $\left([\mathrm{M}+\mathrm{Na}]^{+}, 100 \%\right)$, HRMS $\left(\mathrm{ESI}^{+}\right) \mathrm{C}_{11} \mathrm{H}_{14} \mathrm{~N}_{2} \mathrm{NaO}_{2}{ }^{+}\left([\mathrm{M}+\mathrm{Na}]^{+}\right)$requires 229.0953 ; found 229.1117 .

The instruments used for the data analysis are NMR instruments with models are AVG400/AVF400/AVB400 and IR-Spectrometer used FTS6000/FTS7000 and for mass spectra, we used LC-MS LCT.

\section{RESULTS AND DISCUSSION}

The diethyl malonate was reacted with cyclohex-2-enone, in the presence of anhydrous $\mathrm{K}_{2} \mathrm{CO}_{3}$, in dry $\mathrm{CH}_{2} \mathrm{Cl}_{2}$ heated to reflux for $5 \mathrm{hr}$ and afforded the Michael adduct $\mathbf{1}^{24-28}$ in a very high yield of $82 \%$. This Michael adduct was then readily converted into the corresponding oxime, by using standard conditions $\left(\mathrm{NH}_{2} \mathrm{OH} . \mathrm{HCl}\right.$ and base $\mathrm{Et}_{3} \mathrm{~N}$ in $\mathrm{EtOH}$, heated to reflux for $\left.1 \mathrm{hr}\right)$ and gave oxime 2 again in a very high yield of $94 \%$. The resultant oxime was easily converted into the tosyl oxime ( $p$-toluenesulfonyl chloride and base $\mathrm{Et}_{3} \mathrm{~N}$ in dry $\mathrm{CH}_{2} \mathrm{Cl}_{2}$ ) stirred at r.t. for $2 \mathrm{hr}$ and afforded the tosyl oxime 3 in a high yield of $84 \%$ (Scheme 3). The tosyl oxime 3 was treated with base $\left(\mathrm{NaH}\right.$, in dry THF or anhydrous $\mathrm{CsCO}_{3}$ in dry $\mathrm{CH}_{2} \mathrm{Cl}_{2}$ heated to reflux for $30 \mathrm{~min}$ ) and gave the Beckmann fragmentation product as the substituted cyclopropane ring. The Beckmann fragmentation reaction was repeated with ethyl methyl malonate, tertbutyl ethyl malonate, benzyl ethyl malonate and cyclohex-2-enone in the same sequence as the first Michael addition reaction of dialkyl malonate and cyclohex-2-enone in presence of a mild base as anhydrous $\mathrm{K}_{2} \mathrm{CO}_{3}$ results in adducts in good yield. The same adducts subsequently converted into oximes $\left(\mathrm{NH}_{2} \mathrm{OH} . \mathrm{HCl}\right.$ and base $\mathrm{Et}_{3} \mathrm{~N}$ in EtOH, heated to reflux for $1 \mathrm{hr}$ ) in good yield and further oximes were derivatized with $p$-toluenesulfonyl chloride or 2,4-dinitrofluorobenzene ${ }^{2}$ to tosyloximes or oxime ethers in good yield ${ }^{29-34}$. The tosyloximes or oxime ether on treatment with $\mathrm{NaH}$, in dry THF or anhydrous $\mathrm{CsCO}_{3}$ in dry $\mathrm{CH}_{2} \mathrm{Cl}_{2}$ heated to reflux for 30 min gave good yield of Beckmann fragmentation products as 1,1,2trisubstituted cyclopropanes. (Schemes 4). It was observed that the bulk of the ester group suppressed the attack of nucleophilic carbon leads to the lowering of the yield which was observed in the case of compounds 13 and $17 t$-butyl ester and benzyl ester respectively. Hence compound 5 and compound 9 as diethyl or ethyl methyl ester have moderately high and same yield.

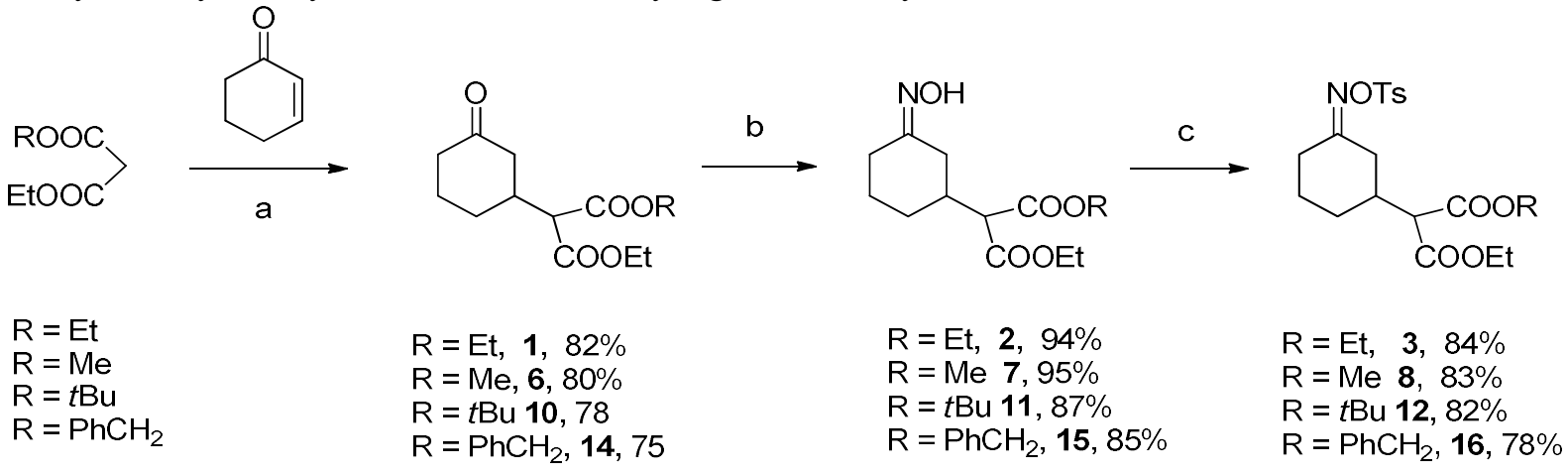

Reagents and conditions (a) anhydrous $\mathrm{K}_{2} \mathrm{CO}_{3}, \mathrm{CH}_{2} \mathrm{Cl}_{2}$, reflux; (b) $\mathrm{NH}_{2} \mathrm{OH} . \mathrm{HCl}, \mathrm{Et}_{3} \mathrm{~N}$; (c) $p \mathrm{TsCl}, \mathrm{Et}_{3} \mathrm{~N}, \mathrm{CH}_{2} \mathrm{Cl}_{2}, \mathrm{rt}_{\text {; }}$ (d) i) $\mathrm{NaH}$, dry THF, reflux, 30 min; ii) anhydrous $\mathrm{CsCO}_{3}, \mathrm{CH}_{2} \mathrm{Cl}_{2}$, reflux.

Scheme-3: Synthesis of Oxime Derivatives

The cyclopropanation ${ }^{35,36}$ through the Beckmann fragmentation reaction was also tested with ethyl 2cyanoacetate, ethyl 2-nitroacetate and cyclohex-2-enone. The Michael addition reaction of ethyl 2cyanoacetate, ethyl 2-nitroacetate and cyclohex-2-enone in presence of mild base as anhydrous $\mathrm{K}_{2} \mathrm{CO}_{3}$ gave good yields. The same adducts then converted into oximes $\left(\mathrm{NH}_{2} \mathrm{OH} . \mathrm{HCl}\right.$ and base $\mathrm{Et}_{3} \mathrm{~N}$ in $\mathrm{EtOH}$, 
RASĀYAN $J$. Chem.

Vol. 13 | No. 4 |2352-2360| October - December | 2020

heated to reflux for $1 \mathrm{hr}$ ) in good yield and further oximes were derivatised with $p$-toluenesulfonyl chloride or 2,4-dinitrofluorobenzene ${ }^{2}$ to tosyloximes or oxime ethers in good yield. The tosyloximes $\mathbf{2 4}$ or oxime ether on treatment with $\mathrm{NaH}$, in dry THF or anhydrous $\mathrm{CsCO}_{3}$ in dry $\mathrm{CH}_{2} \mathrm{Cl}_{2}$ heated to reflux for 30 min gave various results as ethyl 2-cyano-2-(3-((tosyloxy)imino)cyclohexyl)acetate $\mathbf{2 4}$ undergoes Beckmann fragmentation reaction and gave yield $36 \%$ of 25 as ethyl 1-cyano-2-(3cyanopropyl)cyclopropanecarboxylate (1,1,2-trisubstituted cyclopropane) whereas the $\mathbf{2 0}$ tosyloxime (ethyl 2-nitro-2-(3-((tosyloxy)imino) cyclohexyl) acetate) on treatment with $\mathrm{NaH}$, in dry THF or anhydrous $\mathrm{CsCO}_{3}$ in dry $\mathrm{CH}_{2} \mathrm{Cl}_{2}$ heated to reflux did not undergoes Beckmann fragmentation as well as the Beckmann rearrangement reaction and end up with uncharacterized yellow mass. (Schemes-5 and 6).
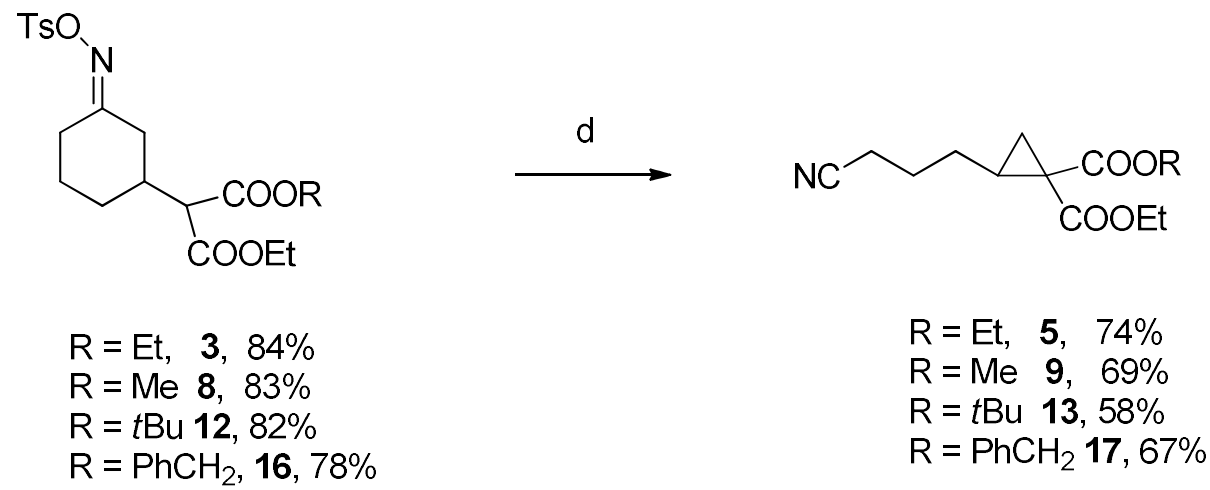

Reagents and conditions (d) i) $\mathrm{NaH}$, dry THF, reflux, 30 min; ii) anhydrous $\mathrm{CsCO}_{3}, \mathrm{CH}_{2} \mathrm{Cl}_{2}$, reflux.

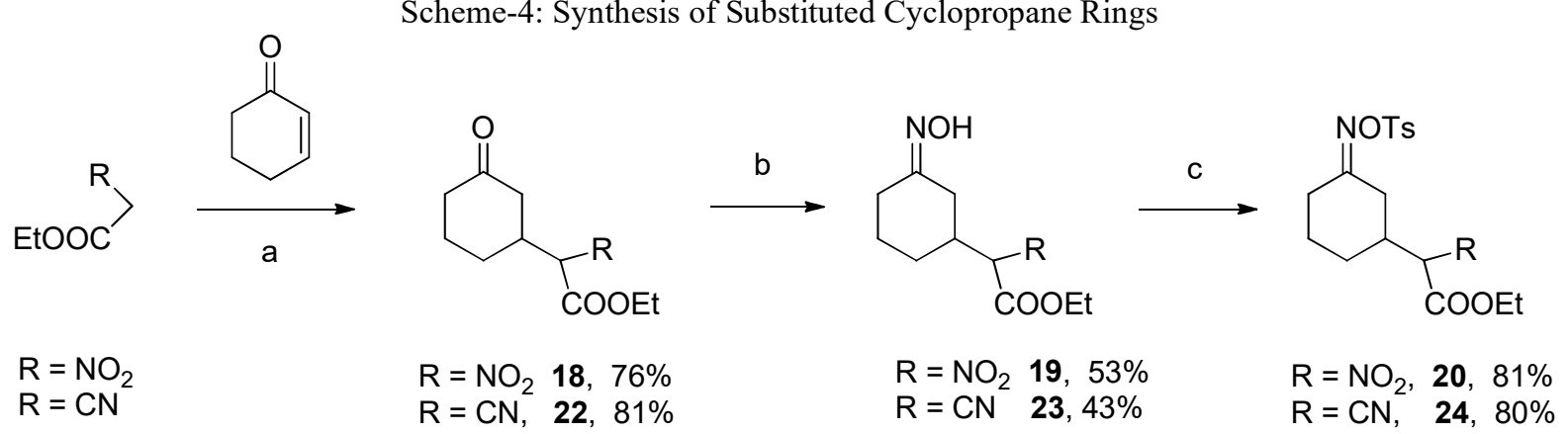

Reagents and conditions (a) anhydrous $\mathrm{K}_{2} \mathrm{CO}_{3}, \mathrm{CH}_{2} \mathrm{Cl}_{2}$, reflux; (b) $\mathrm{NH}_{2} \mathrm{OH} . \mathrm{HCl}, \mathrm{Et}_{3} \mathrm{~N}$; (c) $p \mathrm{TsCl} \mathrm{Et}_{3} \mathrm{~N}, \mathrm{CH}_{2} \mathrm{Cl}_{2}$, rt; (d) i) $\mathrm{NaH}$, dry THF, reflux, $30 \mathrm{~min}$; ii) anhydrous $\mathrm{CsCO}_{3}, \mathrm{CH}_{2} \mathrm{Cl}_{2}$, reflux.

Scheme-5: Oxime Derivatives of Niro, Cyano Adducts<smiles>[R]C(C(=O)OCC)C1CCCC(=NO)C1</smiles>

$$
\begin{aligned}
& \mathrm{R}=\mathrm{NO}_{2}, \quad 20,81 \% \\
& \mathrm{R}=\mathrm{CN}, \quad 24, \quad 80 \%
\end{aligned}
$$

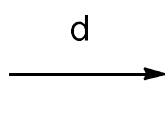<smiles>[R]C1(OCC)CC1CCCCC#N</smiles>

$$
\begin{aligned}
& \mathrm{R}=\mathrm{NO}_{2}, \quad \mathbf{2 1}, \quad \text { didn't work } \\
& \mathrm{R}=\mathrm{CN}, \quad \mathbf{2 5}, \quad 36 \%
\end{aligned}
$$

Reagents and conditions (d) i) $\mathrm{NaH}$, dry THF, reflux, $30 \mathrm{~min}$; ii) anhydrous $\mathrm{CsCO}_{3}, \mathrm{CH}_{2} \mathrm{Cl}_{2}$, reflux. 
RASĀYAN J. Chem.

Vol. 13 | No. 4 |2352-2360| October - December | 2020

\section{CONCLUSION}

In summary unexpected results were obtained while the synthesis of planned anti-Bredt's compounds, when the unknown compounds were characterized and confirmed by NMR and Mass spectroscopy as cyclopropanation through Beckmann Fragmentation as 1,1,2-trisubstituted cyclopropane ring. This methodology was then developed with various substitution patterns as different substituted ester groups and cyano group whereas to incorporate the nitro group using ethyl 2-nitroacetate fails. The methodology developed will be very useful to get quick access to synthesize 1,1,2-trisubstituted cyclopropane ring which is a part of many natural products of high importance.

\section{ACKNOWLEDGEMENT}

I am very much thankful to prof. Mark Moloney, Oxford University, UK for kind support. I also extend my gratitude to my Management PES and principal and my family for continuous help in research. Some of the work has been done at the time of Ph.D and it was sponsored by ministry of Social Justice and Empowerment Government of India.

\section{REFERENCES}

1. R. Keese, Angewandte Chemie International Edition English, 14(9), 528(1975), DOI: 10.1002/anie.197506442.

2. M. A. Drahl, M. Manpadi, L. J. Williams, Angewandte Chemie International Edition English, 52(43), 11222(2013), DOI:10.1002/anie.201209833.

3. Nandkishor Chandan, Amber L. Thompson, Mark G. Moloney, Organic Biomolecular Chemistry, 10, 7863(2012), DOI:10.1039/c2ob26423d.

4. Samuel J. Touchette, Evan M. Dunkley, Leah L. Lowder, Jimmy Wu, Chemical Science, 10, 7812 (2019), DOI:10.1039/c9sc00926d.

5. D. Y. K. Chen, P. H. Pouwer, J. A. Rechird, Chemical Society Review, 41(13), 4631(2012), DOI: $10.1039 / \mathrm{C} 2 \mathrm{CS} 35067 \mathrm{~J}$.

6. M. Nakazaki, K. Naemura, S. Nakahara, Journal of Chemical Society Chemical Communication, 82 (1979), DOI: $10.1039 / \mathrm{C} 39790000082$.

7. L. A. Wessjohann, Chemical Review, 103(4), 1625 (2003), DOI:10.1021/cr0100188.

8. K. Matsumoto, H. Lida, H. Katsura, T. Machiguchi, H. Uekusa, Y. Ohashi, Journal Chemical Society Perkin trans, 1, 2333 (1996), DOI:10.1039/P19960002333.

9. J. O. Reed, W. Cwowaki, Journal of Organic Chemistry, 36(19), 2864(1971), DOI: $10.1021 /$ jo00818a030.

10. M. Toda, Y. Hirata, S. Yamamura, Chemical Communication, 23, 1597(1970), DOI: $10.1039 / \mathrm{C} 29700001597$.

11. H. K. Hall, A. E. Shekeil, Chemical Review, 83, 549(1983), DOI:10.1021/cr00057a004.

12. D. Miljkovic, J. Petrovic, Journal of Organic Chemistry, 42(12), 2101(1977).

13. Poel P. L. Bell, A. Sobolev, J. Wijnberg, A. Groot, Journal of Organic Chemistry, 66(7), 2350 (2001), DOI:10.1021/jo0015568.

14. P. A. Peixoto, D. Y. K. Chen, J. Severin, Organic Letters, 13(21), 5724(2011), DOI: $10.1021 / \mathrm{ol} 202053 \mathrm{~m}$.

15. B. M. Trost, A. Breder, A. W. Franz, Journal of American Chemical Society, 133(13), 4766(2011), DOI: $10.1021 / \mathrm{ja} 200971 \mathrm{v}$.

16. W. A. Donaldson, Tetrahedron, 57(41), 8589(2001).

17. H. Fujioka, M. Miyazaki, H. Kitagawa, T. Yamanaka, K. Takuma. Y. Kita, Journal of Chemical Society, Chemical Communication, 1634 (1993), DOI:10.1039/C39930001634.

18. K. Shea, Tetrahedron, 36, 1683(1980), DOI:10.1016/00404020(80)80067-6.

19. G. Koebrich, Angewandte Chemie International Edition English 12(6), 464(1973), DOI: 10.1002/anie.197304641.

20. M. Nakazaki, K. Naemura, S. Nakahara, Journal of Organic Chemistry, 44(14), 2438(1979), DOI: $10.1021 /$ jo01328a022.

21. S. Ma, S. Yu, S. Yin, Journal of Organic Chemistry, 68(23), 8996 (2003), DOI:10.1021/jo034633i. 
RASĀYAN J. Chem.

Vol. 13 | No. 4 |2352-2360| October - December | 2020

22. Stefan Braese, Hanno Wertal, European Journal of Organic Chemistry, 19, 4167(2005), DOI: $10.1002 /$ ejoc.200500330.

23. Hara Takayoshi, S. Kanai, K. Mori, T. Muzigaki, Journal Organic Chemistry, 71(19), 7455(2006), DOI: $10.1021 /$ jo0614745.

24. A. Westerlund, J. Gras, R. Carlson, Tetrahedron, 57(27), 5879 (2001), DOI:10.1016/S00404020(01)00526-9.

25. John V. Greenhill, Journal of Heterocyclic Chemistry, 29(6), 1375 (1992).

26. Enrique Gomez-Bengoa, J. M. Cuerva, Journal of American Chemical Society, (118)36, 8553(2006).

27. Chen, Donghui, European Journal of Chemistry, 15, 6807 (2002).

28. Magno Agostinho, Shu Kobayashi, Journal of American Chemical Society, 130(8), 2430(2008), DOI: 10.1021/acs.chemrev.7b00692.

29. K. A. Clayton, D. Black, J. Harper, Tetrahedron, 63(43), 10615(2007),DOI: 10.1016/j.tet.2007.08.026.

30. T. D. Wahyuningsih, N. Kumar, D. Black, Tetrahedron, 63, 10613(2007).

31. T. D. Wahyuningsih, K. Pchlk, N. Kumar, D. Black, Tetrahedron, 62, 6343(2006).

32. P. A. Bonvallet, E. M. Todd, Y. S. Kim, R. J. McMahon, Journal of Organic Chemistry, 67(25), 9031 (2002), DOI:10.1021/jo020304z.

33. V. Singh, S. Kanojiya, S. Batra, Tetrahedron, 62(42), 10100(2006), DOI:10.1016/j.tet.2006.08.045.

34. J. A. Milligan, J. P. Phelan, V. C. Polites, C. B. Kelly, G. A. Molander, Organic Letter, 20(21), 6840 (2018), DOI:10.1021/acs.orglett.8b02968.

35. J. P. Phelan, S. B. Lang, J. S. Compton, C. B. Kelly, R. Dykstra, O. Gutierrez, G. A. Molander Journal of American Chemical Society, 140(25), 8037(2018), DOI:10.1021/jacs.8b05243.

36. A. Voituriez, L. E. Zimmer, A. B. Charette, Journal of Organic Chemistry, 75(4), 1244(2010), DOI: 10.1021/jo902618e.

[RJC-5745/2020] 